\title{
Performance assessment of soil moisture sensors under controlled conditions in laboratory setting and recommendations for field deployment
}

\author{
Ana Maria Carmen Ilie *, Cody Goebel, and Tissa Illangasekare \\ Department of Civil and Environmental Engineering, Colorado School of Mines, Golden CO, USA \\ * Correspondence: ailie@mines.edu
}

\begin{abstract}
A three-dimensional intermediate-scale laboratory test system with the ability to control boundary conditions and soil moisture distribution was developed. The developed 3-D soil tank has dimensions of $\mathrm{L}=4.87 \mathrm{~m}, \mathrm{~W}=2.44 \mathrm{~m}$ packed to a depth of $0.40 \mathrm{~m}$. The tank was packed with a heterogeneous configuration using five uniform silica sands with the effective sieve numbers \#70, $\# 16$, \#8, \#12/30, and \#20/30 (Accusands Unimin Corp Ottawa, MN), respectively. Spatial and temporal variations of soil moisture were monitored using 30 embedded soil moisture sensors. Each sensor was individually calibrated for each test sand type to provide unique fitting parameters relating to the sensor's measured voltage to known water content and the data was validated by comparing against a new soil moisture sensor TEROS12. The spatially varying hydraulic properties of the packed sand affected both the water flow and soil moisture dynamics in the tank that was captured by the sensors. Even under such highly controlled test conditions in a laboratory setting, heterogeneities resulting from packing imperfections and compaction introduced some uncertainties to the measurements. These observations suggest the importance of incorporating any available information on the natural heterogeneity when designing sensor deployment strategies in the field.
\end{abstract}

Keywords: soil moisture sensors; soil moisture dynamics; TEROS12 Meter sensors; water content sensor network; water table fluctuations

\section{Introduction}

Accurate soil water content measurements are critical for estimating energy and water balances and understanding chemical and biological processes in the vadose zone, plant root zones, and groundwater. Robinson et al. [1] presented a review of dielectric and electrical conductivity measurements in soils using time domain reflectometry (TDR). Typically, before field deployment, soil water monitoring devices require a rigorous laboratory evaluation and calibration for a range of soil types. Several studies on the evaluation of soil moisture sensors have been reported [2-4]. An evaluation of various ECH2O probe models for soil moisture monitoring showed the sensitivity of sensor readings to supply voltage, temperature, and bulk soil electrical conductivity [5]. Because of the advancing field of wireless sensor technologies, there is an increasing need to deploy distributed soil moisture sensor networks for large spatial coverages. Bogena et al. [5] discussed the need to calibrate the sensors because of their sensitivity to variations of temperature and supply voltage. Sakaki et al. [6,7] provided evidence that sensor-to-sensor differences in the readings were relatively small but not negligible. They proposed a two-point -mixing model to convert sensor readings into soil water content [6]. Recent developments and improvements of $\mathrm{ECH} 2 \mathrm{O}$ soil moisture sensors (Decagon Devices, Inc.) allow for detailed monitoring of soil water content at a relatively low cost both in the laboratory and field. The ECH2O soil moisture sensors comprised of plus and ground prongs where the sensitivity of the plus prong is higher than that of the ground prong. Moreover, the sensor head in which the circuitry is embedded contributes to some of the sensitivity of the measurements [6]. This paper presents preliminary results to test the ability of a soil sensing system to generate data on soil moisture response to changing water table. The experiments focused on 
observing soil moisture patterns in sandy soil and the performance of soil moisture sensors. The goal is to provide some guidance to design the sensor deployment strategies under naturally occurring conditions of soil heterogeneity encountered in the field.

\section{Methods}

\subsection{Experimental Setup}

An intermediate-scale system was developed to test distributed soil moisture sensors under controlled conditions in a laboratory setting. The 3D tank has dimensions of $4.87 \mathrm{~m}$ (length) $\times 2.43 \mathrm{~m}$ (width) $\times 0.40 \mathrm{~m}$ (depth). The tank design allows for the variation of soil moisture by controlling the water table and a rainfall simulator. The water table was controlled using seven constant-head reservoirs with independent boundary heads that allowed for the creation of various flow conditions and dynamic water table configurations within the tank. The system was packed with a heterogeneous configuration using five uniform silica sands with the effective sieve numbers \#8, $\# 12 / 20, \# 16, \# 20 / 30$, and \#70 (Accusands, Unimin Corp., Ottawa, MN), respectively. The sands have porosity values ranging from 0.396 to 0.413 , and a saturated hydraulic conductivity ranging from 1.5 $\mathrm{cm} / \mathrm{s}$ to $0.014 \mathrm{~cm} / \mathrm{s}$. The heterogeneous packing configuration was created by embedding four columns of different sand types in a homogeneous zone with the \#70 sand (Figure 1). The columns were square sections $43.18 \mathrm{~cm} \times 43.18 \mathrm{~cm}$.

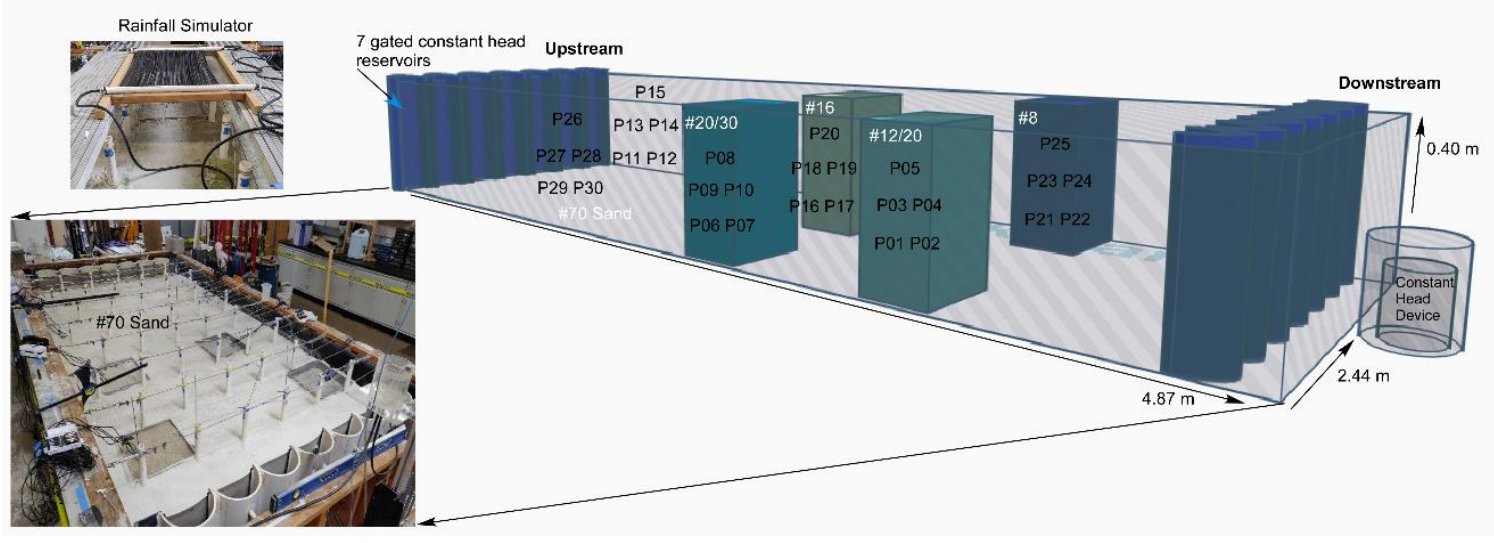

Figure 1. 3D Tank design and soil moisture sensor layout.

A rainfall simulator was designed to control the water flux over the soil surface. The rainfall simulator was made of porous hoses used in irrigation systems. To achieve uniform water distribution on the soil, multiple porous hoses were used, which were further connected to a PVC pipe. The rainfall simulator was connected to a constant head device. Testing showed that it was possible to maintain uniform areal distribution of rainfall across the test area using low water supply pressure to the rainmaker. Thirty soil moisture sensors (Meter Group ECH2O EC5, 5TE, 5TM, ECTM) were calibrated to provide unique fitting parameters relating the measured voltage to the water content of each sensor. Each sensor was calibrated specifically for the five test sands (mesh \#70, \#16, $\# 20 / 30, \# 12 / 20, \# 8$ ) used in creating the heterogeneous packing configuration. Linear regression analysis was used during the calibration process and the coefficient of determination $\left(R^{2}\right)$ was calculated to evaluate the curve fit. The lowest $R^{2}$ value among all the sensors was 0.98 indicating the accuracy of the calibration. Each of the Decagon EM50 data loggers was capable of reading five sensors and hence a total of six data loggers were needed for the 30 sensors. In the sensor calibration experiments, the volumetric moisture content was calculated by dividing the volume of water in the test sample (initial plus added volumes) by the soil volume. The calculated volumetric moisture content was then plotted against the voltage reading of the sensor and a linear regression equation was generated. The intercept and slope obtained of the linear regression equation were used to obtain the calibration curve for each sensor. 
The final instrumentation setup for the tank included six data loggers to monitor 30 soil moisture sensors, where each sensor was assigned a unique ID that included the port number and the data logger (Port 1 to Port 30). Ten soil moisture sensors were distributed in the \#70 sand zone and five sensors were located for each of the remaining four sand types (columns) at a depth of $10 \mathrm{~cm}, 20 \mathrm{~cm}$, and $30 \mathrm{~cm}$ below the surface, respectively. Within each sand type, the sensors at depths of $20 \mathrm{~cm}$ and $30 \mathrm{~cm}$ below the surface were placed $15 \mathrm{~cm}$ apart from each other. Sensor readings were taken once every minute and recorded with the Decagon EM50 data loggers.

\subsection{Design of Experiments}

The 3D-tank setup has the advantage of being able to accurately characterize the heterogeneity through packing with test soils with well-defined properties and to control the boundary and initial conditions that are not possible in field settings. The primary goal of the experiments was to test the ability of a soil sensing system to acquire data of soil moisture response to changing water table and rainfall infiltration. The experiments focused on observing soil moisture patterns in the sandy soil and investigate the performance of soil moisture sensors. Multiple trials were conducted in the experiment to ensure the consistency of the measurements. The water table was raised and lowered using the upstream and the downstream gated reservoirs. The imbibition and drainage phase simulated complex transient soil moisture distribution in the tank to test the ability of the sensors to capture the dynamics.

\section{Results}

This study presents preliminary results from experiments conducted to test the ability of a soil sensing system to generate data on soil moisture response to changing water table and rainfall. A complex transient soil moisture distribution in the tank was observed due to imbibition and drainage which were successfully captured by the sensors.
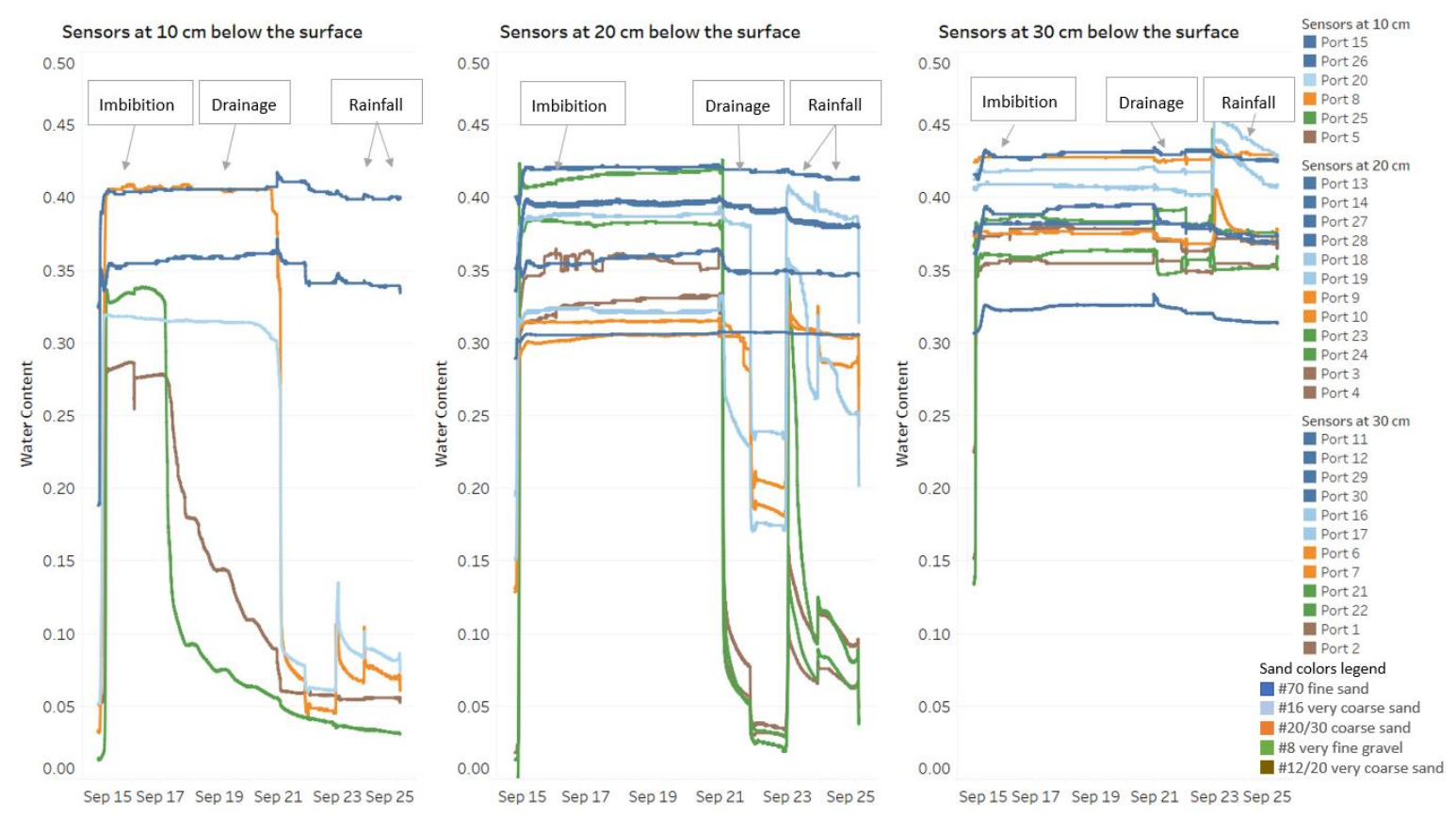

Figure 2. The sensor response to water table fluctuations.

Results from one of the trials are shown in Figure 2, which includes the sensor response plotted against time resulting from the water table fluctuations during imbibition, drainage, and rainfall. Each color represents the type of sand as mentioned in the legend. The plot itself is divided into three sections based on the location of sensor depth below the surface. The water table was raised using upstream reservoirs at a constant level and it can be observed a constant saturation level on sensors 
P11 - P15 and P26 - P30. The water level in the tank was lowered from the right side of the tank (downstream) to bring it back to its initial height, observed by lower saturation levels on sensors P01 - P05, P21 - P25. With a sampling frequency of one minute, no time lag was detected between sensors located next to the left boundary. This was attributed to the faster water propagation, due to higher pressure head, into the porous media which was controlled by the soil permeability. There was no lag observed between sensors that were at the bottom of the tank and located at different distances from the left boundary. On the other hand, there was a significant lag observed between sensors that were vertically aligned in each sand column. For this setup, a delay in saturation levels was also observed between the sensors, where the ones located at the bottom showed higher saturation in comparison to the ones located above. Soil types with different permeability were used to validate the sensor response. As observed from Figure 2, sensors captured soil moisture dynamics depending on the soil type. As expected, a shorter duration of drainage and rainfall process was observed for soils with higher permeability for the sensors located at depth of $10 \mathrm{~cm}$ and $20 \mathrm{~cm}$ from the surface. On the other hand, the processes took longer for the sensors located at $30 \mathrm{~cm}$ from the surface. The low hydraulic conductivity in $\# 70$ - \#20/30 sandy soil attenuated the water flow, which controlled the water level and saturation into the tank.

\subsection{Data Validation}

As a part of the data validation process, soil moisture sensors were compared to the new TEROS12 sensors. Each data logger incorporated five soil moisture sensors located in a sandbox of dimensions $34.3 \mathrm{~cm}$ (length) $\times 47 \mathrm{~cm}$ (width), covered by the same soil type as the one in the 3D tank.

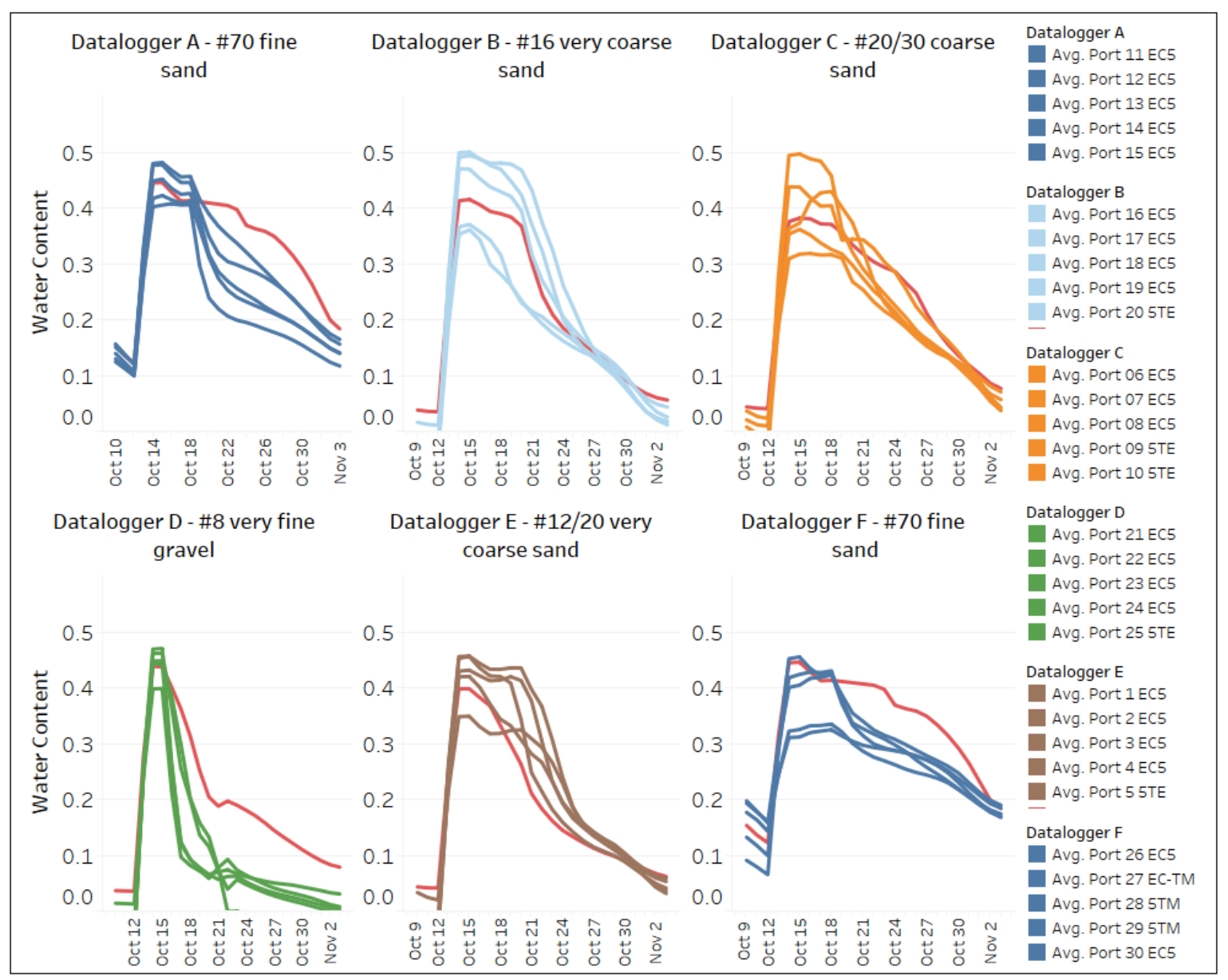

Figure 3. Soil moisture sensor data validation.

Each sandbox was filled with five liters of water which was drained out after two days. The red line in each plot in the grid of Figure 3, indicates the response from TEROS12 and follows a similar 
trend for each set of sensors, thereby validating the sensor response. The higher water content of TEROS12 for data logger A and F can be attributed to the possible interference caused by TEROS12 being located in the middle of the sandbox that was too close to the soil moisture sensors.

\subsection{Statistical Analysis}

Soil moisture sensors performance was evaluated by comparing the response with the new TEROS12 soil moisture sensor. Six statistical parameters, namely correlation coefficient $\left(R^{2}\right)$, mean square error (MSE), root mean square error (RMSE), mean absolute error (MAE), absolute mean bias error (AMBE), and normalized mean square error (NMSE) were calculated as shown in Table 1.

Table 1. Results of performance evaluation between the TEROS12 and EC5 sensors.

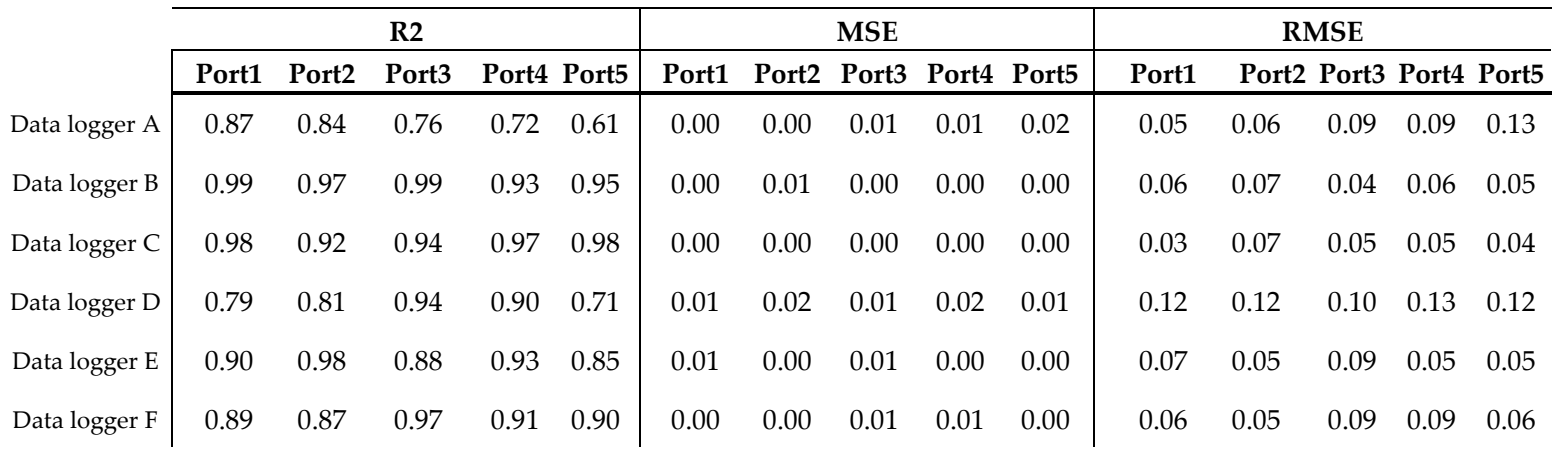

\begin{tabular}{|c|c|c|c|c|c|c|c|c|c|c|c|c|c|c|c|}
\hline & \multicolumn{5}{|c|}{ MAE } & \multicolumn{5}{|c|}{ AMBE } & \multicolumn{5}{|c|}{ NMSE } \\
\hline & Port1 & Port2 & Port3 & Port4 & Port5 & Port1 & Port2 & Port3 & Port4 & Port5 & Port1 & Port2 & Port3 & Port4 & Port5 \\
\hline Data logger A & 0.04 & 0.05 & 0.08 & 0.07 & 0.10 & 0.02 & 0.04 & 0.08 & 0.07 & 0.10 & 0.17 & 0.27 & 0.78 & 0.62 & 1.38 \\
\hline Data logger B & 0.05 & 0.06 & 0.03 & 0.05 & 0.04 & 0.02 & 0.04 & 0.01 & 0.05 & 0.04 & 0.08 & 0.14 & 0.05 & 0.26 & 0.19 \\
\hline Data logger C & 0.03 & 0.06 & 0.04 & 0.05 & 0.04 & 0.00 & 0.01 & 0.02 & 0.05 & 0.04 & 0.05 & 0.15 & 0.10 & 0.28 & 0.12 \\
\hline Data logger D & 0.11 & 0.11 & 0.10 & 0.11 & 0.10 & 0.10 & 0.11 & 0.09 & 0.11 & 0.10 & 0.66 & 0.77 & 0.43 & 0.56 & 1.08 \\
\hline Data logger E & 0.05 & 0.04 & 0.07 & 0.04 & 0.04 & 0.03 & 0.02 & 0.05 & 0.01 & 0.01 & 0.18 & 0.09 & 0.26 & 0.09 & 0.19 \\
\hline Data logger F & 0.05 & 0.04 & 0.08 & 0.08 & 0.05 & 0.05 & 0.03 & 0.07 & 0.08 & 0.05 & 0.32 & 0.35 & 2.65 & 2.30 & 0.29 \\
\hline
\end{tabular}

The results in Table 1 show that the sensors had a good agreement with $\mathrm{R}^{2}$ values ranging between 0.71 and 0.99 . One sensor had an $\mathrm{R}^{2}$ value of 0.61 , possibly due to its proximity to the sandbox wall leading to outliers in the data. An MSE close to zero shows good accuracy between the sensor performance and RMSE, MAE and AMBE indicate a good correlation between the sensors as well. NMSE estimates the overall deviations between the reference (TEROS12) and sensor measurements and is sensitive to extreme values. As an example, for the case of data logger F, multiple outliers were observed, leading to higher values of NMSE.

\section{Conclusions}

The objective of the study that was presented was to evaluate the soil moisture sensors' accuracy in a laboratory setting under different boundary conditions. The soil moisture sensors were calibrated before the experiments for a wide range of soil water content at known temperature conditions. The results from this study will help better understand sensors in a field environment and aid in the interpretation of the data collected. The experiments focused on observing soil moisture patterns and their performance under various imbibition and drainage scenarios. The sensors were able to successfully capture the complex spatial and temporal variations of the soil moisture in the tank. Based on the time-series data, the variations in the specific properties of the sand in the packing led to different saturation levels in the tank. A basic statistical evaluation of the EC5, 5TE, 5TM, EC-TM 
old soil moisture sensors with the new TEROS12 sensors indicates a good agreement for soil water content, proving to be a good candidate for field deployment.

Even in such a controlled environment of this experiment, there was still some heterogeneity within the homogeneous packed zones and some uncertainties in the measurements. However, these preliminary experiments helped to understand the mechanism of soil moisture sensor response and the need to calibrate the sensors to local conditions for better accuracy of the measurements in a field environment. A successful installation in the field requires that the sensor make close contact with the soil. This process becomes challenging for coarse-textured soils where the sensor might lose close contact with the soil itself, thereby influencing the water content measurements. Additionally, sensors should be calibrated to the specific soil, which is going to be located in the field.

Acknowledgments: Funding for this project is provided by NSF and USDA/NIFA.

Conflicts of Interest: The authors declare no conflict of interest. The founding sponsors had no role in the design of the study; in the collection, analyses, or interpretation of data; in the writing of the manuscript, and in the decision to publish the results.

\section{References}

1. Robinson, D.A., Jones, S.B., Wraith, J.A., Or, D., Firedmena, S.P. A review of advances in dielectric and electrical conductivity measurements in soils using time domain reflectometry. Vadose Zone J 2003, 2, 444475 .

2. Leib, B.G., Jabro, J.D., Matthews, G.R. Field evaluation and performance comparison of soil moisture sensors. Soil Science 2003, 168, 396-408.

3. Jones, S.B., Blonquist Jr., J.M., Robinson, D.A., Philip Rasmussen, V., Or, D. Standardizing characterization of electromagnetic water content sensors: part I. Methodology. Vadose Zone J. 2005, 4, 1048-1058.

4. Blonquist Jr., J.M., Jones, S.B., Robinson, D.A., 2005. Standardizing characterization of electromagnetic water content sensors: part 2. Eval. Seven Sens. Syst. Vadose Zone J. 2005, 4, 1059-1069.Author 1, A.; Author 2, B. Book Title, 3rd ed.; Publisher: Publisher Location, Country, 2008; pp. 154-196.

5. Bogena, H.R., Huisman, J.A., Oberdorster, C., Vereecken, H. Evaluation of a low-cost soil water content sensor for wireless network applications. J. Hydrol. 2007, 344, 32-42.

6. Sakaki T, Limsuwat A, Smits KM, Illangasekare TH. Empirical two-point a-mixing model for calibrating the ECH2O EC-5 soil moisture sensor in sands. Water Resour Res 2008, 44, W00D082012.

7. Sakaki, T., Limsuwat, A., and Illangasekare, T.: A Simple Method for Calibrating Dielectric Soil Moisture. Sensors: Laboratory Validation in Sands, Vadose Zone J. 2011, 10, 526-531.

(c) 2020 by the authors; licensee MDPI, Basel, Switzerland. This article is an open-access article distributed under the terms and conditions of the Creative Commons by Attribution (CC-BY) license (http://creativecommons.org/licenses/by/4.0/). 